\title{
BMJ Open Quality Expediting the management of cauda equina syndrome in the emergency department through clinical pathway design
}

\author{
Kevin G Buell, ${ }^{1}$ Sujan Sivasubramaniyam, ${ }^{1}$ Mark Sykes, ${ }^{2}$ Kamran Zafar, ${ }^{3}$ \\ Lucy Bingham, ${ }^{3}$ Anu Mitra ${ }^{3}$
}

To cite: Buell KG,

Sivasubramaniyam S,

Sykes M, et al. Expediting the management of cauda equina syndrome in the emergency department through clinical pathway design. BMJ Open Quality 2019;8:e00597. doi:10.1136/ bmjoq-2018-000597

Received 3 December 2018 Revised 26 September 2019 Accepted 20 0ctober 2019

Check for updates

(c) Author(s) (or their employer(s)) 2019. Re-use permitted under CC BY-NC. No commercial re-use. See rights and permissions. Published by BMJ.

${ }^{1}$ School of Public Health, Imperial College London, London, UK

${ }^{2}$ Department of Medicine, Imperial College London,

London, UK

${ }^{3}$ Departement of Emergency

Medicine, Imperial College

Healthcare NHS Trust, London, UK

Correspondence to

Dr Kevin G Buell;

kevin.g.buell@vumc.org

\section{ABSTRACT}

Introduction Cauda equina syndrome (CES) is a neurosurgical emergency. Early diagnosis with MRI and subsequent surgical decompression surgery can prevent permanent neurological dysfunction. Charing Cross Hospital $(\mathrm{CXH})$ is a tertiary neurosurgical referral centre where in the emergency department (ED), current practice mandated a neurosurgery review prior to requesting MRI.

Hypothesis It was hypothesised that a new clinical pathway, with better coordination from the ED, radiology and neurosurgical teams could reduce the time of presentation to diagnosis or exclusion of CES.

Method Retrospective case-note analysis of patients presenting with back pain to CXH ED over a 3-month period was performed. The primary outcome was the time interval between the patient's arrival to the ED and the MRI preliminary report.

Results The baseline primary outcome was recorded at 8 hours and $16 \min (n=30)$. A new clinical pathway was designed empowering ED senior decision makers to order MRIs prior to neurosurgical review. Two Plan-Do-StudyAct (PDSA) cycles were performed, each measured over a 2-month period. The first PDSA cycle was performed after the pathway was initially launched $(n=17)$, while the second PDSA cycle measured the effect of staff education and active promotion of the pathway $(n=17)$. MRI was requested earlier, waiting and reporting time for MRI were reduced. The exclusion or diagnosis of CES was reduced to 5 hours and 54 min in PDSA 1 and 5 hours $17 \mathrm{~min}$ in PDSA 2 , a $29 \%$ and $36 \%$ reduction $(p=0.048$ and $p=0.012$, respectively).

Conclusion The clinical protocol was a cost-neutral and sustainable intervention that effectively reduced the time taken to diagnose or exclude CES and ED waiting times.

\section{PROBLEM}

Lower back pain is a common medical complaint and its prevalence is rising. ${ }^{1}$

It represents one of the top five reasons why patients attend the emergency department (ED) and accounts for $4.4 \%$ of all ED consultations. ${ }^{2}$ ED physicians must be able to assess lower back pain in a timely fashion, distinguishing benign conditions from those associated with significant morbidity and mortality.
Cauda equina syndrome (CES) is a neurosurgical emergency caused by compression of the sacral and/or lumbar nerve roots, resulting in one or more of the following sequelae: bladder, bowel or sexual dysfunction, decrease perianal sensation, motor or sensory neurological deficit in the lower limbs. ${ }^{3}$ MRI is the gold standard diagnostic test. The Society of British Neurological Surgeons (SBNS) has produced guidelines for the standard of care that is expected in the timely assessment of suspected CES in the ED. They recommend that all EDs have an agreed protocol with their spinal service for the assessment, imaging and referral of patients with suspected CES. ${ }^{4}$ A multidisciplinary and coordinated approach between the $\mathrm{ED}$, radiology and neurosurgical teams is necessary to reduce the time of diagnosis of CES. The Hull Royal Infirmary (UK) has even suggested that this process be standardised through the creation of a national referral pathway. ${ }^{5}$ Early surgical decompression surgery can prevent permanent bladder, bowel and sexual dysfunction ${ }^{6}$ that are associated with delayed diagnosis. ${ }^{7}$

Charing Cross Hospital $(\mathrm{CXH})$ is a tertiary neurosurgical referral centre in London, UK, and has a busy ED, seeing approximately 120000 patients per year. The agreed practice instructed ED physicians to refer patients with suspected CES to neurosurgery for further evaluation, including the decision regarding the need for MRI. Senior ED physicians were unable to request MRI without prior neurosurgical review.

It was hypothesised that better coordination from the ED, radiology and neurosurgical teams could reduce the time of presentation to diagnosis or exclusion of CES. A team composed of ED senior house officers, registrars and consultants was created to lead a quality improvement project (QIP) evaluating 
our current practice for patients with suspected CES in the CXH ED. The team would identify factors that caused delay in diagnosis of CES and if applicable, design a new, sustainable, and cost-neutral intervention to expedite the management of suspected CES. The SMART aim of this QIP was to reduce the time to diagnosis of CES by $25 \%$ within 6 months. All data relevant to the study are included in the article.

\section{BACKGROUND}

The prevalence of CES is low, with estimates ranging between 1:33 000 and 1:100 000 inhabitants. ${ }^{8}$ Large central lumbar disc herniation at $\mathrm{L} 4 / 5$ or $\mathrm{L} 5 / \mathrm{S} 1$ is the most common cause of CES, ${ }^{9}$ although rarer causes such as spinal trauma, neoplasm, spinal abscesses and multilevel stenosis can also be responsible. Red flag symptoms, such a motor weakness, loss of sensation in the legs or peri-anal area, decreased or absent deep tendon reflexes, urinary incontinence or retention, and loss of anal tone should raise suspicion for CES. These signs help distinguish CES from other causes of lower back pain.

Female gender and age above 30 years have been identified as two risk factors associated with $\mathrm{CES}^{10}$ and it is felt that most patients affected are young to middle age. ${ }^{11}$ Delayed and/or misdiagnosis can cause irreversible sphincter and sexual dysfunction, a devastating outcome on a patient's personal and social life. It equally has high medico-legal ramifications. In the UK, the Medical Defence Union paid out $£ 6.7$ million in compensation over a 5-year period, with an average of $£ 336000$ per claim based at 2003 rates. ${ }^{12}$

The urgency of decompression surgery and how timing affects clinical outcomes has predominantly been studied in retrospective studies, as prospective randomised controlled trial are not ethically feasible. In those with preserved or partially preserved bladder function, early decompression within 24 hours increases the likelihood of bladder function recovery. ${ }^{13}$ DeLong et als metaanalyses showed that urinary retention is correlated to worst clinical outcomes, and in these cases, the effect of the timing of decompression surgery is less certain. ${ }^{14}$ However, since establishing the exact onset of symptoms in patients is difficult, the SBNS advocates that surgery for CES should be performed as soon as possible. ${ }^{4} \mathrm{~A}$ 4-year study at St. George's Hospital neurosurgical centre in London retrospectively investigated factors leading to delayed treatment of CES. It concluded that delayed treatment was most frequently caused by a delay in making the diagnosis. ${ }^{15}$

In summary, there is evidence in the literature and recommendations from the SBNS to suggest that EDs should strive for early diagnosis of CES to decrease severe adverse patient outcomes. Clinical pathways and protocols have been successfully designed in the ED to streamline and improve patient care in sepsis. ${ }^{16}{ }^{17}$ As such, a clinical pathway was felt to be the most cost effective, proven and tailored clinical intervention for the QIP.

\section{BASELINE MEASUREMENT}

The project started in September 2016 and assessed the baseline performance of the CXH ED in managing patients with suspected CES. Using electronic online medical records, data were retrospectively collected for a 3-month period (15 June-15 September 2016) for patients presenting to the CXH ED with back pain. Data were collected for the following variables: patient demographics, arrival time to the $\mathrm{ED}$, presenting complaint, findings on the lower limb neurology examination performed by the ED physician and neurosurgical physician, time the MRI was requested, time the MRI took place, time of MRI report. Data were collected onto a Microsoft Excel (Microsoft 2011) spread sheet.

A total of 393 patients presented for evaluation of lower back pain. Patients in which CES was not the primary differential diagnosis or those transferred directly to the neurosurgery service directly from another hospital were excluded. Thirty patients were referred by their general practitioner (GP) or self-presented, underwent emergency MRI with the intent of diagnosing CES. Eleven were male, 19 were female and the average age was 47 years. The presence or absence of five objective findings documented in the lower limb neurology examination were reviewed: decreased lower limb power, reduced/ absent lower limb sensation, decreased/absent deep tendon reflexes, decreased/absent perianal sensation and decreased/absent anal tone. Comparing the documentation of the examination findings between the ED and neurosurgery physicians, there was an $81 \%$ agreement between the two. All patients assessed by the ED were referred to neurosurgery (NS) for further management. The NS evaluation agreed in $85 \%$ of cases referred that CES was the most likely diagnosis. All but three ED physicians were senior house officers and all assessments made by the neurosurgery team were registrars.

The average time needed to exclude or diagnose CES, measured by the time interval between the patient's ED arrival and release of the MRI preliminary report, was 8 hours and $16 \mathrm{~min}$. This was categorised into the three steps: 3 hours and 3 min between the patient presenting to the ED and MRI request, 3 hours and 37 min between the request and scan taking place and 1 hour and 36 min for the release the preliminary report (table 1 ). The

Table 1 Baseline measurements of the average time needed to exclude or diagnose cauda equina syndrome before pathway implementation

\begin{tabular}{llll}
\hline & \multicolumn{3}{l}{ Components of the patient journey } \\
\cline { 2 - 4 } & $\begin{array}{l}\text { ED arrival } \\
\text { to MRI } \\
\text { request }\end{array}$ & $\begin{array}{l}\text { MRI } \\
\text { request to } \\
\text { MRI scan }\end{array}$ & $\begin{array}{l}\text { MRI scan to } \\
\text { provisional } \\
\text { report }\end{array}$ \\
\hline $\begin{array}{l}\text { Mean time (min) } \\
\text { Proportion of total } \\
\text { patient journey (\%) }\end{array}$ & 183 & 217 & 96 \\
\hline
\end{tabular}

ED, emergency department. 
average length of stay in the ED was 5 hours and 48 min. The UK's national 4 hours ED waiting time target was breached in $63 \%$ of cases.

\section{DESIGN}

The SMART aim was to decrease the length of time needed to exclude or diagnose CES by $25 \%$, in patients who self-presented or were referred by GPs to the ED. The goal was to be achieved within a 6 months period.

The baseline data were presented to the ED, neurosurgery and radiology consultants to ensure engagement of the key multidisciplinary stakeholders. It was agreed that the current hospital practice for the management of CES needed revision. The views of 36 ED staff members, including nurses, charge nurses, junior doctors, registrars and consultants, were opportunistically surveyed to assess the biggest barriers in assessing CES in a timely manner. Arranging a neurosurgical review and obtaining an MRI were identified as the two biggest impediments creating delays. Eighty-six per cent of ED staff agreed that a clinical pathway would facilitate their assessment of CES. The staff wanted the pathway to improve the collaboration between the ED, neurosurgery and radiology teams, specifically placing the MRI request earlier in the patient journey. The need for teaching was also highlighted.

As such, the principal intervention for the QIP was the implementation of a new clinical pathway that would address the barriers identified by our staff: improved work synergy between the emergency, neurosurgery and radiology departments resulting in MRIs being ordered

Imperial College Healthcare W/HS

Suspected Cauda Equina Syndrome (CES) Pathway

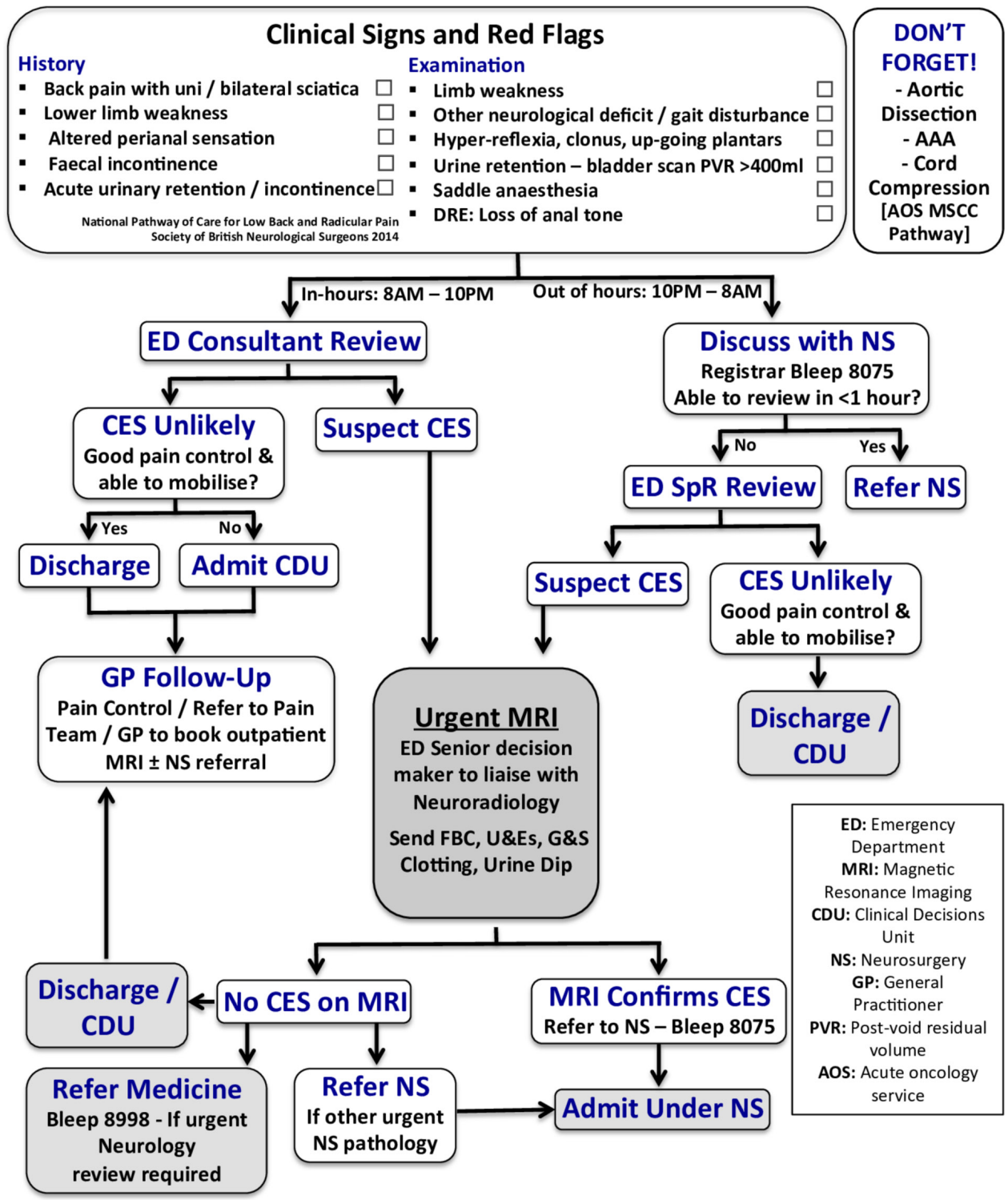

Figure 1 Clinical pathway for the assessment of CES in the Charing Cross Hospital (CXH) ED. NHS, National Health Service. 
earlier in the patient journey. Figure 1 shows the pathway, which was approved by the hospital's managerial board. It ensured early ED consultant assessment of patients suspected of CES and empowered ED consultants to order MRIs prior to neurosurgery review. They would then communicate their assessment over the phone to the radiology team. The patient would be referred to neurosurgery on the basis of the MRI result. At the times when the ED consultants were not on-duty, the pathway operated similarly with the ED registrar taking the lead, with the exception that if the neurosurgeon could review the patient within the hour. The primary outcome to assess the success of the clinical pathway was the time interval between the patient's arrival to the ED and the MRI preliminary report.

\section{STRATEGY}

\section{PDSA cycle 1}

The clinical pathway was introduced to all hospital staff members involved in the care of patients with suspected CES. It was presented to the ED staff during a mandatory weekly training session and to the neurosurgery and radiology departments during an internal meeting in July 2017. The pathway was updated on the guidelines section of the ED intranet page.

\section{PDSA cycle 2}

Interventions in the second PDSA cycle were designed in accordance to the feedback received by our staff, obtained through semistructured opportunistic interviews. The need for the pathway to be more readily visible and a demand for further teaching on the CES project were highlighted to the QIP team. There were multiple interventions performed simultaneously for the second PDSA cycle designed to increase the visibility of the pathway. Posters of the pathway were displayed in all key work areas in the ED, neurosurgery and radiology departments. An online PDF version of the pathway was used as a screensaver on the projector used in morning handover and a brief reminder about the pathway was incorporated into the daily morning handover. Teaching about CES and the pathway was added to the mandatory 2-day induction of new ED junior doctors. An ED senior house officer reviewed the assessment of lower back pain, CES and our pathway with the ED nurses as part of their mandatory teaching sessions. It was also presented to local GPs at a regional primary care engagement seminar.

\section{RESULTS}

The same endpoints used in the baseline measurements were recollected over an 8-week period, 20 July-14 September 2017, after the interventions of PDSA cycle 1 (introduction of the clinical pathway) and over a 12-week period, 2 November-25 January 2018, after the interventions of PDSA cycle 2 (improved visibility of the clinical pathway) to measure the effectiveness of our

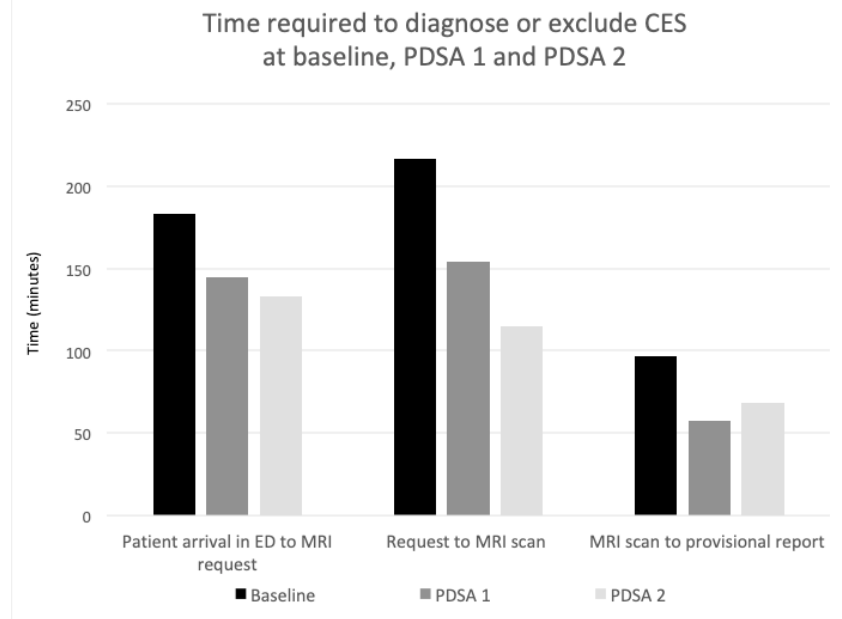

Figure 2 Bar chart categorising the time interval between the patient's arrival to the emergency department (ED) and MRI preliminary report at baseline, PDSA 1 and PDSA 2. CES, cauda equina syndrome.

interventions. Seventeen patients were included in each cycle of data collection.

The primary outcome was the time interval between the patient's arrival to the ED and the MRI preliminary report. It was measured at 5 hours and 54 min in PDSA 1 and 5 hours $17 \mathrm{~min}$ in PDSA 2. Compared with a baseline of 8 hours $16 \mathrm{~min}$, this represents a $29 \%$ and $36 \%$ reduction in time. The improved performance was statistically significant, $\mathrm{p}=0.048$ in PDSA cycle 1 and $\mathrm{p}=0.012$ in PDSA cycle 2 (figure 2). Between PDSA 1 and PDSA 2, there was a statistically non-significant $10 \%$ reduction $(\mathrm{p}=0.362)$.

The length of stay in the ED was 3 hours and 5 min in PDSA 1 and 3 hours $18 \mathrm{~min}$ in PDSA 2. Although the average time in the ED decreased from baseline to PDSA $1,65 \%$ of patients still breached the national 4 hours waiting time standard. In PDSA 2, 29\% of patients breached, which was statistically significant compared with baseline $(p=0.03)$.

The overall proportion of patients who underwent MRI for the investigation of suspected CES remained constant between $10 \%$ and $12 \%$ at baseline, PDSA 1 and PDSA 2, irrespective of whether MRI were ordered by neurosurgery or ED physicians.

\section{LESSONS AND LIMITATIONS}

We believe that our accomplishment stemmed from involving key stakeholders from each department and engaging them in the design of the pathway. This QIP demonstrated good concordance between the ED and neurosurgical assessments of patients (alleviating concerns about patient safety) and proposed a cost neutral intervention, through a simple re-organisation of the available resources. ED physicians did not order more MRIs than neurosurgeons. Furthermore, the pathway proved to be cost effective for the Trust, as there were fewer ED breaches in the second PDSA cycle. The 
support of ED nurses and junior doctors was also critical, as they were responsible for initiating the pathway. Questionnaires for feedback promoted education and engagement required for the project's success. Therefore, our success is most likely a reflection that this change was planned across all departments and was well received among all levels of staffing.

As with all change, there were limitations encountered. A slow staff engagement was the biggest barrier at the start. To overcome this, multiple discussions were held with nurses, senior house officers, registrars and consultants during working hours to reiterate the importance of the pathway for patient care and encourage its use. If we could repeat this QIP, we would increase the time interval between designing the pathway and implementing it to allow for more education and awareness of the pathway prior to launching it. We learnt that for a pathway to be utilised, it must be readily visible and available. Feedback highlighted that using the ED website and initial teaching was not sufficient in raising awareness. Consequently, our second PDSA cycle was focused around promoting the pathway through a variety of other methods previously described.

Although our QIP did result in a reduction in the time needed to diagnose CES in the ED, we did not evaluate whether this translated to better patient outcomes from earlier surgical decompression surgery. The decision to proceed with emergency surgery overnight versus waiting until the morning, where more senior staff members are present, was beyond the scope of this project. Despite the SBNS recommending immediate surgery, some professional opinions have suggested that as long as surgery is performed within 48 hours of symptom onset, ${ }^{18}$ delaying surgery until the morning may be a safer alternative. ${ }^{9}$ As emergency physicians, the decision to proceed with surgery overnight is outside the limit of our practice. Equally, the neurosurgery team did not want this decision incorporated into the pathway, as it was dependent on multiple patient, physician and staffing factors. As CES is a condition where delaying surgery certainly does not improve outcomes, we believe a reduction in the time of diagnosis is an improvement in the care delivered to our patients. It gives the neurosurgeons the best possible tools to make a definitive treatment decision.

Subjectively, waiting for MRI was a source of great frustration and anxiety for patients. We hoped that this pathway would alleviate this problem and shorten the patient journey. Patient questionnaires used at baseline and with each PDSA cycle could have been used to assess whether a reduction in the waiting time for MRI led to greater patient satisfaction in the care they received.

Finally, the number of patient cases was low with 30 patients at baseline and 17 with each subsequent PDSA cycle. Although our results reached statistical significance, a greater number of patients would provide more robust evidence on the effectiveness of our clinical pathway. To recruit more patients, the duration of each PDSA cycle would have had to be extended, as CES remains a rare diagnosis in patients presenting with lower back pain.

\section{CONCLUSIONS}

This QIP successfully achieved its aim by reducing the time interval from presentation to the exclusion or diagnosis of CES by an average 2 hours and $59 \mathrm{~min}$, a $36 \%$ reduction from baseline. Originally designed to be cost neutral, patients were less likely to breach the national 4 hours ED waiting time which resulted in cost saving for the hospital. The change has been fully integrated and sustained into practice in all three departments involved. Following the success of the project, the pathway has been rolled out to St. Mary's Hospital, our sister hospital within the Imperial National Health Service Healthcare Trust. Our hope is that other EDs will draw from our experience and the lessons we have learnt through this project.

Acknowledgements The authors would like to thank the hard-working staff of the CXH ED for their individual contributions that helped launch, improve and sustain this project into the care we deliver for our patients.

Contributors KGB, SS, MS, KZ, LB and AM contributed in the study concept and design. KGB, MS and SS retrospectively collected the data. KGB and SS performed the statistical analysis and interpretation of the data. KGB drafted the first version of the manuscript. KGB, SS and MS critically revised the manuscript for intellectual content. KGB, SS, MS, KZ, LB and AM reviewed and approved the final version of the manuscript for submission. LB and AM supervised the project as senior authors.

Funding The authors have not declared a specific grant for this research from any funding agency in the public, commercial or not-for-profit sectors.

Competing interests None declared.

Patient consent for publication Not required.

Provenance and peer review Not commissioned; externally peer reviewed.

Open access This is an open access article distributed in accordance with the Creative Commons Attribution Non Commercial (CC BY-NC 4.0) license, which permits others to distribute, remix, adapt, build upon this work non-commercially, and license their derivative works on different terms, provided the original work is properly cited, appropriate credit is given, any changes made indicated, and the use is non-commercial. See: http://creativecommons.org/licenses/by-nc/4.0/.

\section{REFERENCES}

1 Hoy D, Bain C, Williams G, et al. A systematic review of the global prevalence of low back pain. Arthritis Rheum 2012;64:2028-37.

2 Edwards J, Hayden J, Asbridge M, et al. Prevalence of low back pain in emergency settings: a systematic review and meta-analysis. BMC Musculoskelet Disord 2017;18:143.

3 Fraser S, Roberts L, Murphy E. Cauda equina syndrome: a literature review of its definition and clinical presentation. Arch Phys Med Rehabil 2009;90:1964-8.

4 Germon T, Ahuja S, Casey ATH, et al. British association of spine surgeons standards of care for cauda equina syndrome. Spine $J$ 2015;15:S2-4.

5 Hussain MM, Razak AA, Hassan SS, et al. Time to implement a national referral pathway for suspected cauda equina syndrome: review and outcome of 250 referrals. Br J Neurosurg 2018;32:264-8.

6 Korse NS, Pijpers JA, van Zwet E, et al. Cauda equina syndrome: presentation, outcome, and predictors with focus on micturition, defecation, and sexual dysfunction. Eur Spine J 2017;26:894-904.

7 Kennedy JG, Soffe KE, McGrath A, et al. Predictors of outcome in cauda equina syndrome. Eur Spine J 1999;8:317-22.

8 Dias ALN, Araújo FFde, Cristante AF, et al. Epidemiology of cauda equina syndrome. what changed until 2015. Rev Bras Ortop 2018;53:107-12.

9 Lavy C, James A, Wilson-MacDonald J, et al. Cauda equina syndrome. BMJ 2009;338:b936-7. 
10 Schoenfeld AJ. Incidence and epidemiology of cauda equina syndrome: a review of 976 patients from a complete American population. Spine J 2012;12:S100-1.

11 Fuso FAF, Dias ALN, Letaif OB, et al. Epidemiological study of cauda equina syndrome. Acta Ortop Bras 2013;21:159-62.

12 Gardner A, Gardner E, Morley T. Cauda equina syndrome: a review of the current clinical and medico-legal position. Eur Spine $J$ 2011;20:690-7.

13 Todd NV. Cauda equina syndrome: the timing of surgery probably does influence outcome. Br J Neurosurg 2005;19:301-6.

14 DeLong WB, Polissar N, Neradilek B. Timing of surgery in cauda equina syndrome with urinary retention: meta-analysis of observational studies. J Neurosurg 2008;8:305-20.
15 Jalloh I, Minhas P. Delays in the treatment of cauda equina syndrome due to its variable clinical features in patients presenting to the emergency department. Emerg Med J 2007;24:33-4.

16 Thursky K, Lingaratnam S, Jayarajan J, et al. Implementation of a whole of hospital sepsis clinical pathway in a cancer Hospital: impact on sepsis management, outcomes and costs. BMJ Open Qual 2018;7:e000355.

17 Pinnington S, Atterton B, Ingleby S. Making the journey safe: recognising and responding to severe sepsis in accident and emergency. BMJ Qual Improv Rep 2016;5:u210706.w4335.

18 Ahn UM, Ahn NU, Buchowski JM, et al. Cauda equina syndrome secondary to lumbar disc herniation: a meta-analysis of surgical outcomes. Spine 2000;25:1515-22. 